\title{
Rational Expectations Equilibrium: An Alternative Approach*
}

\author{
Lawrence E. Blume \\ Department of Economics, University of Michigan, \\ Ann Arbor, Michigan 48109 \\ AND \\ DAVID EASLey \\ Department of Economics, Cornell University, \\ Ihaca, New York 14853 \\ Received June 29, 1982; revised June 6, 1983

\begin{abstract}
We study a dynamic market process in which traders condition their beliefs about payoff-relevant parameters on past endogenously generated market data and current exogenous data. We say that a market process is informative if the beliefs of traders who receive only endogenously generated market data converge almost surely to the true parameter value. Our main result is that under standard regularity hypotheses, the generic market process is informative. We define an equilibrium as the limit points of the market process. Journal of Economic Literature Classification Numbers: 021.026. c 1984 Academic Press. Inc.
\end{abstract}

\section{INTRODUCTION}

There are few general results on the statistical properties of marketclearing prices in economies with incomplete markets and asymmetrically informed traders. One result is that if the amount of private information is sufficiently small, then equilibrium prices typically will be sufficient statistics for that information (Allen [2,3], Radner [20]). Another result is that if the amount of private information is sufficiently "large," then equilibrium prices typically will not be sufficient (Jordan and Radner [18], Jordan [16]).

These results present several problems. When equilibrium prices are

* This work has greatly benefitted from seminars at Harvard, NYU, Stonybrook, U. C. Santa Barbara, and at the NBER/CEME Decentralization Conference at Stanford in May 1981. Conversations with Bruce Hill and Jim Jordan were especially helpful. This research was begun while the first author was visiting the Center for Mathematical Studies in Economics and Management Science at Northwestern University. Support for both authors from the National Science Foundation is gratefully acknowledged. 
sufficient statistics, the equilibrium cannot be implemented by collecting information on excess demands alone. This is the content of the famous "Beja paradox." (For a discussion, see Jordan and Radner [19].) When the amount of private information is large, not only will prices not be sufficient, but the equilibrium has so far been shown to exist only with constructions leading to a mathematically perverse price statistic-one discontinuous on a dense set of signals (Jordan [17]). This particular view of the market is not compelling.

We believe these implementation and existence problems arise because static Walrasian models are ill-suited to the study of statistical properties of equilibrium. Prices have too much to do when statistical inference and market clearing occur simultaneously.

In our view, markets evolve over time as traders condition their behavior on past endogenously generated market data and current exogenous data. Traders rationally use whatever information they have. However, if they were able to extract information from current endogenous data, the process which implements market equilibria would require revelation of entire excess demand functions. Rather than require the existence of institutions that accomplish this revelation, we find it more natural to assume that institutions preclude conditioning behavior on current equilibrium data.

We model the dynamic market process as a recursive system. Our rationality hypothesis is that traders make correct use of exogenous data and predetermined market data. Just as current market data may not be sufficient for current private information (Jordan [16]), past market data may not be sufficient for past private information. Sufficiency, however, is only an optimality property of a statistic. Past market data may contain useful information without being sufficient for past private information. Estimates of payoff-relevant parameters from endogenously generated market data may ultimately be just as accurate as parameter estimates from exogenous data.

We use the tools of statistical decision theory to characterize the limit behavior of the stochastic market process. We say that a market process is informative if the beliefs of traders who do not receive exogenous information about payoff-relevant parameters converge almost surely to certain knowledge of the true parameter value. In other words, a market process is informative if Bayes estimates (from market data alone) of the payoffrelevant parameters are, in the language of statistical decision theory, consistent. Our main result is that under standard regularity hypotheses, the generic market process is informative.

Learning behavior in our model can be thought of as a Nash equilibrium in learning strategies, as in Townsend [22]. Thus the model presented here can be described as a rational learning model, in stark contrast to our earlier, boundedly rational model of learning behavior (Blume and Easley [9]). A class of examples exploiting similar decision theoretic tools has been 
discussed by Bray and Kreps [11]. Recursive sequential equilibrium models have recently been studied by Hellwig [14].

The model is presented in Section 2, and general dynamics are treated in Section 3. Limiting behavior is discussed in Section 4. The informativeness of the sequence of market data is analyzed in Section 5. Section 6 contains the proof of our major theorem. Summaries and conjectures are collected in Section 7.

\section{THE MODEL}

Our intent is to construct the simplest possible model to illustrate our alternative concept of rational expectations equilibrium. In our model each generation of traders lives for one period in which they purchase and consume a bundle of goods. The utility value of these bundles is random and unknown ex ante to some traders, the uninformed, and is known to others, the informed. The parameters describing the utility of the bundles are distributed according to one of a finite number of distributions. Uninformed traders want to learn which distribution the values come from. Thus an uninformed trader born in period $t$ uses public market data from previous periods, which reflects to some extent the information of past informed traders, to estimate which distribution is correct. We identify conditions under which Bayes estimates, from market data, of the parameter indexing distributions are consistent.

We study a sequence of temporary equilibria for an exchange economy with $L$ perishable goods. Let $\{1, \ldots, i, \ldots, I\}$ denote the set of trader types. All traders have consumption set $R_{++}^{L}$, and traders of type $i$ have an endowment in each period of $\omega_{i} \in R_{++}^{L}{ }^{1}$ Also given is a set $S \subset R^{n}$ of payoff-relevant parameters where $S$ is a compact set with non-empty interior. In each period the single trader of each type selects a consumption bundle to maximize his expected utility. Utility satisfies the following assumptions:

A.1. $u_{i}: R_{++}^{L} \times S \rightarrow R^{1}$ is $C^{2}$ with the usual boundary condition that for all $s \in S$ closures in $R^{L}$ of level sets do not intersect the boundary of $R_{++}^{L}$.

A.2. For all $(x, s) \in R_{++}^{L} \times S, \quad D_{x} u_{i}(x, s) \in R_{++}^{L}, \quad$ and $D_{x}^{2} u_{i}(x, s) \in R^{L^{2}}$ is negative definite.

It follows that for any Borel probability measure on $S$, expected utility is $C^{2}$ on $R_{++}^{L}$, satisfies the boundary condition of A.1, and satisfies A.2.

${ }^{1} R_{++}^{L}=\left\{x \in R^{L}: x_{j}>0\right.$ for all $\left.j\right\}$. We use $C^{P}, p \geqslant 0$, as an abbreviation for $C^{P}\left(R^{m}, R^{n}\right)$, the set of $p$ times continuously differentiable functions from $R^{m}$ to $R^{n}$, when $R^{m}$ and $R^{n}$ are unambiguous. For a function $f \in C^{2}\left(R^{m}, R^{1}\right), D f(x)$ denotes the $m$-vector of first partial derivatives of $f$ and $D^{2} f(x)$ denotes the $m \times m$ matix of second partial derivatives of $f$. 
The parameter $s \in S$ at time $t$ comes from the realization of an i.i.d. stochastic process $\left\{s_{t}\right\}_{t=1}^{\infty}$. The distribution of $s_{t}$ is unknown, but each trader correctly believes it to be contained in the set $\left\{\mu_{\theta}: \theta \in \Theta\right\}$ where each $\mu_{\theta}$ is a Borel probability measure on $S$ and $\Theta$ is a set of parameters.

A.3 The cardinality $|\Theta|$ of $\Theta$ is finite.

Any trader of type $i$ starts with prior $\beta_{i}$ on $\Theta$ and then given his information he uses Bayes rule to compute a posterior. As $\Theta$ is finite, beliefs at time $t$ can be represented by vectors $\beta_{i t}=\left(\beta_{i t}(\theta)\right)_{\theta \in \Theta}$ in the non-negative unit simplex $A^{|\theta|-1}$. No trader could learn that $\theta \in \Theta$ is true if he believes it to be impossible a priori, so we assume:

A.4 For all $i, \beta_{i}(\theta)>0$ for all $\theta \in \Theta$.

Let $E$ denote the set of all vectors $e=\left(u_{1}, \ldots, u_{l}\right)$ of utility functions satisfying A.1 and A.2. A sequence $\left\{e_{n}\right\}_{n=1}^{\infty}$ converges to limit $e$ if and only if for all $i$ the maps $u_{i n}(x, s), D_{x} u_{i n}(x, s)$, and $D_{x}^{2} u_{i n}(x, s)$ all converge uniformly to $u_{i}(x, s), D_{x} u_{i}(x, s)$, and $D_{x}^{2} u_{i}(x, s)$, respectively. Evidently if a sequence $\left\{e_{n}\right\}_{n=1}^{\infty}$ converges to limit $e$ in this sense, then for any vector of probability distributions $\left(v_{1}, \ldots, v_{I}\right)$ on $S$, the corresponding sequence $\left(\int u_{1 n}(x, s) d v_{1}, \ldots, \int u_{I n}(x, s) d v_{I}\right)$ of expected utility functions converges in the topology of uniform $C^{2}$ convergence on compact subsets of $R_{++}^{L}$ to $\left.\left(u_{1}(x, s) d v_{1}, \ldots, u_{I}(x, s) d v_{t}\right)\right)^{2}$ This fact will be used without comment in the sequel.

In Section 5 we fix the endowment allocation and prove that certain statements are generic. This is to say that certain statements hold on open and dense sets of $E$.

\section{DYNAMICS}

At time $t$ one trader of each type is born. As each trader lives for only one period, his objective is to maximize the expected utility of his consumption at $t$. Trader types $i=1, \ldots, I_{1}$ are informed of the value of $s_{t}$ in each period $t$. These informed traders ignore market data as it contains no additional information. Trader types $i=I_{1}+1, \ldots, I$ are not informed of the value of any $s_{t}$. Hence, at $t$ an uninformed trader of type $i$ uses past market data to revise his prior $\beta_{i}$ to a posterior $\beta_{i t}$.

At time $t$ the informed trader of type $i$ solves the maximization problem

$$
\begin{gathered}
\operatorname{MAX} u_{i}\left(x_{i t}, s_{t}\right) \\
\text { s.t. } p_{t} \cdot\left(x_{i t}-\omega_{i}\right) \leqslant 0, \quad x_{i t} \in R_{++}^{L}
\end{gathered}
$$

${ }^{2}$ This follows immediately by differentiating under the integral. 
where $p_{t}$ denotes a price vector in the unit simplex $\Delta^{L-1}$. It is a standard exercise to show that the excess demand for informed trader $i$ is a $C^{1}$ function $z_{i}: \Delta^{L-1} \times S \rightarrow R^{L-1}$ satisfying all the usual conditions necessary for equilibrium analysis.

At time $t$ the uninformed trader of type $i$ solves the maximization problem

$$
\begin{gathered}
\operatorname{MAX} \int u_{i}\left(x_{i t}, s_{t}\right) d v_{i t} \\
\text { s.t. } p_{t} \cdot\left(x_{i t}-\omega_{i}\right) \leqslant 0, \quad x_{i t} \in R_{++}^{L}
\end{gathered}
$$

where $v_{i t}$ is the posterior predicted distribution of $s_{t}$ given trader $i$ 's observations. The posterior predicted distribution of $s_{t}$ is easily derivable from trader $i$ 's posterior beliefs about $\theta$. The posterior beliefs of $i$ are a vector $\beta_{i t} \in \Delta^{|\theta|-1}$ so

$$
v_{i t}=\frac{\searrow}{\theta \in \Theta} \beta_{i t}(\theta) \mu(0)
$$

It is a standard exercise to show that excess demand for uninformed trader $i$ in period $t$ is a $C^{1}$ function $z_{i}: \Delta^{L-1} \times \Delta^{|\theta|-1} \rightarrow R^{1-1}$ which satisfies all the usual conditions necessary for equilibrium analysis.

Let $\bar{\Delta}=\prod_{i=I_{1}+1}^{I} \Delta^{|\Theta|-1}$. Aggregate excess demand at time $t$ is

$$
z: \Delta^{L-1} \times \bar{\Delta} \times S \rightarrow R^{L-1}
$$

defined as

$$
z\left(p, \beta_{I_{1}+1}, \ldots, \beta_{1}, s\right)=\sum_{i=1}^{I_{1}} z_{i}(p, s)+\sum_{I_{1}+1}^{I} z_{i}\left(p, \beta_{i}\right) .
$$

It is straightforward to guarantee that equilibrium always exists and that the equilibrium price correspondence

$$
F: \bar{\Delta} \times S \rightarrow \Delta^{L-1}
$$

defined by

$$
F\left(\beta_{I_{1}+1}, \ldots, \beta_{I}, s\right)=\left\{p \in \Delta^{L-1}: z\left(p, \beta_{I_{1}+1}, \ldots, \beta_{I}, s\right)=0\right\}
$$

is upper-hemicontinuous.

The dynamics of the market process are determined by the evolution of posterior beliefs once we specify how prices are actually determined. Naturally the particular equilibrium price vector which emerges from the set of all possible market clearing prices will be determined by the institutions which implement market processes. We suppose only that price deter- 
mination can be represented as a measurable selection $f$ from the correspondence $F$. A measurable selection always exists (Hildenbrand [15]) and our results are independent of the selection chosen.

Thus dynamics for our model will be completely specified once we specify the information structure. We assume that uninformed traders observe only publically available past market data and that this data is only market prices. ${ }^{3}$ In order to simplify notation we denote this information by $M$ rather than by $\Delta^{L-1}$.

Let $M_{\infty}=X_{\tau=1}^{\infty} M$. Let $\tilde{M}_{t}$ denote $\sigma$-field of Borel measurable events in $M_{\infty}$ that are observable at or before time $t$; i.e., $A \in \tilde{M}_{t}$ iff $A=B \times X_{\tau=t+1}^{\infty} M$, where $B$ is measurable in $X_{\tau=1}^{t} M$. Let $\tilde{M}_{\infty_{t}}=V_{\tau=1}^{\infty} \tilde{M}_{t}$. Then $\left\{\tilde{M}_{t}\right\}_{t=1}^{\infty}$ is a sequence of $\sigma$-fields adapted to $\tilde{M}_{\infty}$ on $M_{\infty}$. Uninformed traders at time $t$ condition on events in $\tilde{M}_{t-1}$. This is the recursion assumption. Uninformed traders only condition on predetermined endogenous variables. (In period 1, uninformed traders use their prior predicted distributions.)

We say that the stochastic process of market data is informative if uninformed traders can ultimately learn the true $\theta$.

DEFINITION 1. The stochastic process of market data $\left\{p_{t}\right\}_{t=1}^{\infty}$ is informative if for each $i=I_{1}+1, \ldots, I \lim _{t \rightarrow \infty} E\left\{\beta_{i}(\theta) \mid \tilde{M}_{t}\right\}=1$ a.s. when the true parameter is $\theta$.

Note that the distribution of the process $\left\{p_{t}\right\}_{t=1}^{\infty}$ can be derived by substituting back through $f$ and the Bayes revision rules. This process is very complicated. For example, typically it will not be Markov. Nonetheless the limit behavior of this process can be characterized because it is driven by conditional expectations, to which Martingale theory can be applied.

The dynamics of the market process are as follows. First, the new informed traders receive observations of the payoff-relevant parameters. Next, the new uninformed traders revise the prior for their type based on past market data. Then trade occurs. Finally, each trader consumes and dies. Then the process repeats itself with a new generation of traders.

\section{The Limit ECONOMY}

The process of market prices is driven by the expectations formation mechanism. Thus suppose $\bar{\theta}$ is the true parameter value, and that $A$ is an event in $\tilde{M}_{t}$. Then

$$
\operatorname{Pr}_{\bar{\theta}}(A)=\operatorname{Pr}_{\bar{\theta}}\left\{f\left(\beta_{I_{1}+1, \tau}, \ldots, \beta_{I, \tau}, s_{\tau}\right)_{\tau=1}^{t} \in B\right\}, \quad \text { where } A=B \times X_{\tau=t+1}^{\infty} M .
$$

${ }^{3}$ If the information set contains more than market prices any results guaranteeing convergence of beliefs to certain knowledge of the relevant parameter value will still hold. 
In principle this can be computed in a straightforward manner from $\mu_{\bar{\theta}}$ and the Bayesian updating formulae. Our assumption that uninformed traders are Bayesians is in effect an assumption that they all know $\operatorname{Pr}_{\theta}$ for each $\theta \in \Theta$.

Consider an uninformed trader of type $i$ with prior probability distribution $\beta_{i}$ and let $\tilde{\Theta}$ denote the $\sigma$-field of measurable events in $\Theta$. (We have assumed that $\Theta$ is finite, so $\widetilde{\Theta}$ is the power set of $\Theta$. But this discussion is fairly general. For example, it suffices to require that $(\Theta, \tilde{\Theta})$ be a complete seperable metric space.) Then trader $i$ 's beliefs give rise to a probability measure on the measurable space $\left(\Theta \times \tilde{M}_{\infty}, \sigma\left(\widetilde{\Theta} \times \tilde{M}_{\infty}\right)\right)$ defined as the extension of

$$
\operatorname{Pr}_{i}\{A \times B\}=\int_{B} \operatorname{Pr}_{\theta}\{A\} d \beta_{i}
$$

for $A \in \tilde{M}_{\infty}, B \in \widetilde{\Theta}$. (Of course when $\Theta$ is not finite, the measurability of the map $\theta \rightarrow \operatorname{Pr}_{\theta}(A)$ is required, but this is easy to show.) Then the posterior probability trader $i$ assigns to event $B \in \tilde{\Theta}$ at time $t$ is

$$
E\left\{1_{B \times M_{x}} \mid \bar{M}_{t-1}\right\}
$$

where $\bar{M}_{t-1}$ is the product of $\tilde{M}_{t-1}$ and the trivial $\sigma$-field $\{\varnothing, \Theta\}$, and the expectation is taken with respect to the probability distribution $\operatorname{Pr}_{i}{ }^{4}$ The sequence of $\sigma$-fields $\{\bar{M}\}_{t=1}^{\infty}$ is increasing, and so the sequence of conditional expectations $\left\{E\left\{1_{B \times M} \mid \bar{M}_{t}\right\}\right\}_{t=1}^{\infty}$ is a uniformly bounded Martingale. Thus the Martingale convergence theorem applies. The sequence of random variables $\left\{E\left\{1_{B \times M} \mid \bar{M}_{t}\right\}\right\}_{t=1}^{\infty}$ converges almost surely to a $V_{t=1}^{\infty} \bar{M}_{t}$-measurable limit random variable, which is the limit posterior probability of the event $B \in \tilde{\Theta}$. It is an easy exercise to extend this in the following manner:

Define the period $t$ posterior distribution for traders of type $i$ by

$$
\beta_{t}(B)=E\left\{1_{B \times M} \mid \bar{M}_{t-1}\right\}
$$

Then $\beta_{t}$ is a random variable whose values are probability distributions on $(\Theta, \tilde{\Theta})$. This sequence of random variables converges almost surely in the topology of weak convergence to a $V_{t=1}^{\infty} \bar{M}_{t}$-measurable limit random variable whose values are probability distributions.

To sum up, let $P(\Theta, \widetilde{\Theta})$ denote the set of all probability measures on the measurable space $(\Theta, \widetilde{\Theta})$, with the topology of weak convergence. Posterior distributions are $P(\Theta, \widetilde{\Theta})$-valued random variables.

THEOREM 1. Let $\left\{\beta_{i t}\right\}_{t=1}^{\infty}$ be type i's sequence of posterior beliefs. Then there exists a $P(\Theta, \tilde{\mathcal{\Theta}})$-valued variable $\beta_{i \infty \infty}$ such that $\lim _{t \rightarrow \infty} \beta_{i t}=\beta_{i \infty}$ a.s. $\mu_{\theta}$.

Proof. See Appendix.

${ }^{4} 1_{F}$ takes the value 1 on $E$ and 0 elsewhere. 
Let $Q=X_{\tau=1}^{\infty} S$ be the space of sequences of signals drawn from $S$ and let $\tilde{Q}$ be the $\sigma$-field on $Q$. The sequence of random variables $\left\{\left(\beta_{I_{1}+1, t}, \ldots, \beta_{I, t}\right)\right\}_{t=1}^{\infty}$ is defined on $(Q, \tilde{Q})$, where $q \in Q$ is drawn according to the i.i.d. process whose one-dimensional distribution is $\mu_{\theta}$, and converges almost surely to $\left(\beta_{I_{1}+1, \infty}, \ldots, \beta_{I, \infty}\right)$. Since market data in any period depends only on the informed's signal in that period and the expectations that uninformed traders hold when they enter the market and since expectations converge almost surely, there is a sense in which market data converges. Let $d$ be the metric on $M \times M$.

Lemma 1. $\operatorname{Inf}\left\{d\left(p_{t}, y_{t}\right): y_{t} \in F\left(\beta_{I_{1}+1, \infty}, \ldots, \beta_{t, \infty}, s_{t}\right)\right\}$ converges to 0 almost surely.

Proof. The correspondence $F$ is non-empty and compact valued, so $\inf \left\{d\left(p_{t}, y_{t}\right): y_{t} \in F\left(\beta_{I_{1}+1, \infty}, \ldots, \beta_{I, \infty}, s_{t}\right)\right\}$ is well defined. Choose a point $q \in Q$ such that $\left\{\beta_{I_{1}+1, t}, \ldots, \beta_{t . t}\right\}_{t=1}^{\infty}$ converges. Then inf $\left\{d\left(p_{t}, y_{t}\right)\right.$ : $\left.y_{t} \in F\left(\beta_{I_{1}+1, \infty}, \ldots, \beta_{1, \infty}, s_{t}\right)\right\}$ converges to 0 as a consequence of the upper hemi-continuity of $F$.

Q.E.D.

Since convergence of expectations is an event in the tail $\sigma$ field of the equilibrium price process, it is necessary to characterize the limit behavior of equilibrium prices. Lemma 1 provides a useful characterization. The sequence $\left\{p_{t}\right\}_{t=1}^{\infty}$ is informative if traders learn the true $\theta$ with probability 1 , and so a sequence $\left\{p_{t}\right\}_{t=1}^{\infty}$ of random variables is informative if with probability 1 a trader can tell from which distribution $\theta$ was drawn. In the next section this is done by studying the limit sets of prices identified in Lemma 1. When uninformed traders' expectations converge to the vector $\left(\beta_{i_{1}+1, \infty}, \ldots, \beta_{1, \infty}\right)$ they all know this limit vector as they all use the Bayesian updating formula and they all start with positive prior probability on every $\theta$. Then almost surely the only possible limit sets of data are those that arise from the i.i.d. stochastic process $\mu_{\theta} \circ f^{-1}\left(\beta_{I_{1}+1, \infty}, \ldots, \beta_{I, \infty}\right)$. If this process is informative, then, as the process of market data converges to this process, uninformed traders will be able to learn $\theta$.

\section{Information Contained in Market Data}

Now we look at the information carried by the stochastic process of market prices $\left\{p_{t}\right\}_{t=1}^{\infty}$. In effect, uninformed traders at time $t$ use $\left\{p_{\tau}\right\}_{\tau=1}^{t-1}$ in an attempt to learn $\theta$. The following theorem gives conditions under which Bayes estimates of $\theta$ from this data are consistent.

THEOREM 2. Suppose A.1-A.4 are satisfied. There exists a residual set 
of economies $C \subset E$ such that for each $e \in C$ there exists an open and dense set of vectors of measures in $\Pi_{\theta \in \Theta} P(S, \tilde{S})$ such that for any $\left(\mu_{1}, \ldots, \mu_{|\theta|}\right)$ in this set prices are informative for the economy.

\section{Proof of THEOREM 2}

The proof of this theorem involves applying the techniques of static rational expectations analysis to the limit economy which is, as we have seen, an i.i.d. stochastic process. If Bayes estimates of $\theta$ from the limit price process are consistent for each possible specification of limit posteriors for the uninformed traders, then limit beliefs are correct. (This follows because convergence of Bayes estimates of $\theta$ from prices is a property of the tail $\sigma$ field, and we have seen that on the tail $\sigma$-field the stochastic price process agrees almost surely with one of the limit i.i.d. processes described in Section 4.)

Suppose that the limit i.i.d. process has distribution $v_{\theta}(\bar{\beta})$ when the parameter is $\theta$ and the limit vector of beliefs is $\bar{\beta} \in \bar{A}$. Then $\theta$ can be estimated consistently if and only if the $v_{\theta}(\bar{\beta})$ distributions are all different (Doob [12], Schwartz [21]). If $f: \Delta \times \bar{S} \rightarrow \Delta^{L-1}$ is the selection from the equilibrium price correspondence, then

$$
v_{\theta}(\bar{\beta})=\mu_{\theta} \circ f^{-1}(\bar{\beta}, \cdot)
$$

This is to say, $v_{\theta}(\bar{\beta})$ is the distribution under $\mu_{\theta}$ of the random variable $f(\bar{\beta}, \cdot): S \rightarrow \Delta^{L-1}$.

In order to prove our results for all possible selections from the equilibrium correspondence it will be necessary to work directly with distributions of the equilibrium correspondence. Define

$$
\mu \circ F^{-1}(\bar{\beta}, \cdot)=\left\{\mu \circ f^{-1}(\bar{\beta}, \cdot): f \text { is a measurable selection from } F\right\} .
$$

LEMma 2. If $F: \bar{\Delta} \times S \rightarrow \Delta^{L-1}$ is u.h.c., then the correspondence $\Gamma: P(S, \tilde{S}) \times \bar{\Delta} \rightarrow P\left(\Delta^{L-1}, \tilde{A}^{L-1}\right)$, defined such that $\Gamma(\mu, \bar{\beta})=\mu \circ F^{-1}(\bar{\beta}, \cdot)$, is u.h.c.

Proof. Blume [7, Theorem 3.1].

We first show that for all $e \in E$, the set of measures $\left(\mu_{1}, \ldots, \mu_{|\theta|}\right) \in \Pi_{\theta \in \Theta} P(S, \widetilde{S})$ such that $\Gamma\left(\mu_{\theta}, \bar{\beta}\right) \cap \Gamma\left(\mu_{\theta^{\prime}}, \bar{\beta}\right)=\varnothing$ for all $\bar{\beta} \in \bar{\Delta}$ and $\theta \neq \theta^{\prime}$ is open. If this is true the property that prices are informative is open.

LEMma 3. The set of all measures $\left(\mu_{1}, \ldots, \mu_{|\theta|}\right) \in \Pi_{\theta \in \Theta} P(S, \tilde{S})$ such that $U_{\bar{\beta} \in \Delta} \Gamma\left(\mu_{\theta}, \bar{\beta}\right) \cap \Gamma\left(\mu_{\theta^{\prime}}, \bar{\beta}\right)=\varnothing$ for all $\theta \neq \theta^{\prime}$ is open. 
Proof. It suffices to establish the lemma for any pair $\theta \neq \theta^{\prime}$. Define $A: P(S, \tilde{S}) \rightarrow \bar{\Delta} \times P\left(\Delta^{L-1}, \tilde{\Delta}^{L-1}\right)$ such that $\Lambda(\mu)=\left\{\left(\bar{\beta}, \mu \circ F^{-1}(\bar{\beta}, \cdot)\right): \bar{\beta} \in \bar{\Delta}\right\}$. It follows from Lemma 2 and the compactness of $P\left(\Delta^{L-1}, \tilde{A}^{L-1}\right)$ that $A$ is u.h.c. Note that $U_{\bar{\beta} \in \bar{J}} \Gamma\left(\mu_{1}, \bar{\beta}\right) \cap \Gamma\left(\mu_{2}, \bar{\beta}\right)=\varnothing$ is true if and only if $A\left(\mu_{1}\right) \cap$ $\Lambda\left(\mu_{2}\right)=\varnothing$. Now the $\Lambda\left(\mu_{i}\right)$ are compact sets (since $A$ is u.h.c. and $\bar{\Delta} \times P\left(\Lambda^{L-1}, \tilde{\Delta}^{L-1}\right)$ is compact) and so there exists open sets $A_{i} \supset \Lambda\left(\mu_{i}\right)$ such that $A_{1} \cap A_{2}=\varnothing$. Let $N_{i}=\left\{\mu: A(\mu) \subset A_{i}\right\}$. Since $A$ is u.h.c. each $N_{i}$ is open, and if $\left(\mu_{1}, \mu_{2}\right) \in N_{1} \times N_{2}, A\left(\mu_{1}\right) \cap \Lambda\left(\mu_{2}\right)=\varnothing$.

Q.E.D.

The only thing left to prove is the density statement in Theorem 2 . We first show that if the measures $\left\{\mu_{\theta}: \theta \in \Theta\right\}$ have finite, sufficiently large, disjoint supports, then the limit sets described in Lemma 2 are disjoint. If this is the case, the asymptotic behavior of the equilibrium price sequence clearly identifies $\theta$. We then use this result to establish that prices in the limit economy will reveal $\theta$ for a dense set of measures in $\Pi_{\theta \in \Theta} P(S, \tilde{S})$ for any member of a residual set of economies.

Let $k$ be an arbitrary integer greater than $\left(I-I_{1}\right)(|\Theta|-1) /(L-1)$, and let $\left(s_{1}^{1}, \ldots, s_{k}^{|\theta|}\right)$ be any $k|\Theta|$ different vectors from $S$. Suppose that $\mu_{1}$ has for its support the first $k$ vectors, $\mu_{2}$ the second $k$ vectors, etc. Then supp $\mu_{\theta} \cap$ $\operatorname{supp} \mu_{\theta^{\prime}}=\varnothing$ for $\theta=\theta^{\prime}$. Definc $s_{1}^{\theta}, \ldots, s_{k}^{\theta}$ to be the vectors in supp $\mu_{\theta}$. Let $\bar{\beta}$ denote the limit beliefs of the uniformed. A necessary condition for any two different parameters $\theta$ and $\theta^{\prime}$ to be indistinguishable in the limit economy is that there exists a permutation $\pi$ of the integrers $1, \ldots, k$ such that

$$
\begin{aligned}
& z\left(p_{1}, \bar{\beta}, s_{\pi(1)}^{\theta}\right)=0 \\
& \vdots \\
& z\left(p_{k}, \bar{\beta}, s_{\pi(k)}^{\theta}\right)=0 \\
& z\left(q_{1}, \bar{\beta}, s_{1}^{\theta^{\prime}}\right)=0 \\
& \vdots \\
& z\left(q_{k}, \bar{\beta}, s_{k}^{\theta^{\prime}}\right)=0 \\
& p_{1}-q_{1}=0 \\
& \vdots \\
& p_{k}-q_{k}=0 .
\end{aligned}
$$

Call the left-hand side of this equation $H\left(p_{1}, \ldots, p_{k}, q_{1}, \ldots, q_{k}, \bar{\beta}\right)$. Then $H: \prod_{j=1}^{2 k} \Delta^{L-1} \times \bar{\Delta} \rightarrow R^{3 k(L-1)}$. It is easy to see that $H$ is a differentiable map. If (1) has a solution, then it is possible to select from $F$ so that $\operatorname{supp} \mu_{\theta} \circ f^{-1}(\bar{\beta}, \cdot)=\operatorname{supp} \mu_{\theta}, \circ f^{-1}(\bar{\beta}, \cdot)$.

Lemma 4. If $k>\left(I-I_{1}\right)(|\Theta|-1) /(L-1)$, then there exists an open 
and dense set of economies $E\left(s_{1}^{1}, \ldots, s_{k}^{|\Theta|}\right) \subset E$ such that (1) has no solution for any permutation $\pi$, any $\theta, \theta^{\prime} \in \Theta$, and any $\bar{\beta} \in \bar{\Delta}$.

Proof. The idea is to use Allen's [1] idea of log-linear perturbations of traders' utility functions. Consider trader 1 , an informed observer. Define the smooth function $\delta^{\theta i}: S \rightarrow R$ for $i=1, \ldots, k$ and $\theta \in \Theta$ such that $\delta^{\theta i}\left(s_{i}^{\theta}\right)=\varepsilon^{\theta i}$ and $\delta^{\theta i}\left(s_{j}^{\theta^{\prime}}\right)=0$ for $i \neq j$ or $\theta \neq \theta^{\prime}$. Let $\alpha_{1}^{\theta i}, \ldots, \alpha_{L}^{\theta i}$ be real numbers such that $\sum_{l=1}^{L} \alpha_{l}^{\theta i} \leqslant 1$. Let $\alpha=\left(\alpha_{l}^{11}, \ldots, \alpha_{L}^{|\theta| k}\right)$ and $\varepsilon=\left(\varepsilon^{11}, \ldots, \varepsilon^{|\theta| k}\right)$. Now consider, for trader $i$,

$$
u_{i}(x, s, \alpha, \varepsilon)=u_{i}(x, s)+\sum_{i, \theta}^{\prime} \delta^{\theta i}(s) \Sigma_{l=1}^{\prime} \alpha_{l}^{\theta i} \ln x_{l} .
$$

This utility function satisfies A.1 and A.2, and has the effect of introducing a different $\log$-linear perturbation for each $s_{i}^{\theta}$. Our method now follows Allen [1] and Radner [20]. Define, in the obvious way, $H(p, q, \bar{\beta}, \alpha)$. Computing,

$$
D H=\left[\begin{array}{ccccc}
A & A^{\prime} & A^{\prime \prime} & 0 & 0 \\
0 & 0 & B^{\prime \prime} & B & B^{\prime} \\
I & 0 & 0 & -I & 0
\end{array}\right]
$$

where $A$ and $B$ are $k(L-1) \times k(L-1)$ matrices representing the derivatives $D_{p} z\left(p, \bar{\beta}, s_{\pi(i)}^{\boldsymbol{\theta}}\right)$ and $D_{p} z\left(p, \bar{\beta}, s_{i}^{\boldsymbol{\theta}^{\prime}}\right)$, respectively; $A^{\prime \prime}$ and $B^{\prime \prime}$ are $k(L-1) \times\left(I-I_{1}\right)(|\Theta|-1)$ matrices representing derivatives with respect to $\bar{\beta}$; and $A^{\prime}$ and $B^{\prime}$ are $k(L-1) \times k L$ matrices representing derivatives with respect to the $\alpha_{1}^{\theta i}$ and $\alpha_{l}^{\theta i^{\prime}}$, respectively. The key fact, due to Allen [1], is that $A^{\prime}$ and $B^{\prime}$ are surjective. This implies the surjectivity of $D H$, and so $H \pi\{0\}$. The solution set has codimension $3 k(L-1)$, and so it is a manifold of dimension $2 k(L-1)+\left(I-I_{1}\right)(|\Theta|-1)+2 k L-3 k(L-1)=\left(I-I_{1}\right)$ $(|\Theta|-1)-k(L-1)+2 k l$. The $2 k L$ corresponds to the $a$ perturbations. If $k>\left(I-I_{1}\right)(|\Theta|-1) /(L-1)$, then the dimension is less than $2 k L$. Thus the projection of the solution set onto the space of $\alpha$ perturbations is lower dimensional. This establishes density of the economies for which (1) has no solution for any $\bar{\beta}$, and openness is obvious. Intersecting open and dense sets over a finite number of permutations $\pi$ and a finite number of pairs $\theta \neq \theta^{\prime}$ leaves an open and dense set, which proves the lemma.

Q.E.D.

To complete the proof of the theorem we need only construct a dense set of measures in $\prod_{\theta \in \Theta} P(S, \tilde{S})$ like those of Lemma 3 . Let $D(k)$ denote a countable and dense set of vectors $\left\{\left(s_{1}^{1}, \ldots, s_{k}^{|\Theta|}\right) \in \prod_{j=1}^{k|\theta|} S: s_{i}^{\theta} \neq s_{j}^{\theta^{\prime}}\right.$ for $i \neq j$ or $\left.\theta \neq \theta^{\prime}\right\}$. From Lemma $4,\left(s_{1}^{1}, \ldots, s_{k}^{|\Theta|}\right) \in D(k)$ implies $E\left(s_{1}^{1}, \ldots, s_{k}^{|\Theta|}\right)$ is open and dense if $k$ is sufficiently large. Define

$$
E(k)=\bigcap_{\left(s_{1}^{1}, \ldots, s_{k}^{|\theta|} \in D(k)\right.} E\left(s_{1}^{1}, \ldots, s_{1}^{|\Theta|}\right) .
$$


This set is residual. Let $C=\bigcap_{k>\bar{k}} E(k)$ where $\bar{k}=\left(I-I_{1}\right)(|\Theta|-1) /(L-1)$. This set is residual, and for any economy $e \in C$ and limit beliefs $\bar{\beta}$ the limit economy has distinct i.i.d. price distributions for each $\theta \in \Theta$ when the parametrization $\left\{\mu_{\theta}: \theta \in \Theta\right\}$ is such that $\left(\operatorname{supp} \mu_{1}, \ldots\right.$, supp $\left.\mu_{|\theta|}\right) \in D(k)$ for any $k$ sufficiently large. This set of such parametrizations is dense in $\prod_{\theta \in \Theta} P(S, \tilde{S})$ (Billingsley [6]), and this completes the proof of the theorem.

\section{CONCLUSION}

In a world where market institutions preclude traders from conditioning their expectations on current endogenous market data, past market data may contain useful information. Uninformed traders may use it to infer something that the informed know or are learning. The model we have constructed was designed to illustrate our alternative approach to rational expectations equilibria in the simplest possible context. Our model can clearly be enriched to include other phenomena and stronger theorems may be found with a deeper probabilistic analysis of the sample paths of market data.

In particular, our method of analysis can be used to ask whether or not the uninformed can learn the true structural model. Suppose $\theta$ is a parameter describing some aspect of the informed payoffs which is also relevant for the uninformed as it enters the structural model. Then our analysis would be applied to give conditions under which the uninformed could learn $\theta$. The technique is to show that in the limit economy prices identify $\theta$ for an open and dense set of economies (i.e., perturb the informed trader's payoffs for values of $\theta$, rather than for values of $s$ ) and then argue, as in the text, that since the limit $\sigma$-field identifies $\theta$, market data is informative. Bray and Kreps [11] provide a closely related analysis of this problem.

The other problem suggested by our analysis lies in the nature of the intertemporal link. In our model the only connection between different dates is the dependence of posterior beliefs upon past observations. In particular we allow no durable goods, no savings, and no money. If these phenomena are allowed, the limit economy becomes much harder to characterize and so our methods may not suffice. One possibility, though, is to find conditions which give rise to turnpike properties of equilibrium. Steady-state savings or consumption plans may tie down the limit economy sufficiently for our methods to succeed. Recent work by Bewley [5] may provide guidance in this direction.

There is a more important question suggested by our results. The least appealing aspect of our definition of rationality is the amount of knowledge we require of each trader. A theory of market evolution which requires all traders to have full knowledge of the structure of the economy is at least imperfect, if not misguided. In a trivial sense we can allow for learning about 
the structure of the economy, but this only pushes the common knowledge assumption one level deeper. At some level the structure must be fixed, exactly as the space of types is known to all players in Harsanyi's [13] theory of games of incomplete information. The obvious question is. To what extent can this assumption be relaxed? The negative results of Blume and Easley [9] suggest what can happen when traders arbitrarily misspecify the likelihood function for market data.

We leave the reader to choose between two conflicting interpretations of our results. First, our Theorem 2 provides a basis for belief in rational expectations equilibria in the long run, because the structrure of the economy can be learned by Bayesians from endogenous market data. Second, the hypotheses of our Theorem 2 are unbearably strong, but the alternative is the negative results of our earlier paper, and so there is no reason to believe that market forces drive the economy to a rational expectations equilibrium.

\section{APPENDIX}

Proof of Theorem 1. Define $\mu_{t}(B)=E\left\{1_{B \times M} \mid \bar{M}_{t}\right\}$ for all $B \in \tilde{\Theta}$. So defined, $\mu_{t}$ is a probability measure on the measurable space $(\Theta, \widetilde{\Theta})$. Since $1_{B \times M}$ is an indicator function, $\sup _{t} E\left\{1_{B \times M^{2}} \mid \bar{M}_{t}\right\}<1$ a.s., so the sequence $\left\{\mu_{t}(B)\right\}_{t=1}^{\infty}$ is a square-integrable martingale. From the martingale convergence theorem, the limit random variable $\mu_{\infty}(B)=\lim _{t \rightarrow \infty} \mu_{t}(B)$ exists almost surely. Since $(\Theta, \widetilde{\Theta})$ is Polish, there exists a countable collection of sets $\left\{B_{j}\right\}_{j=1}^{\infty}$ which form a convergence-determining class for the topology of weak convergence on $(\Theta, \tilde{\Theta})$ (see $\left[6\right.$, p. 14]). As $\left\{B_{j}\right\}_{j-1}^{\mathcal{O}_{j}}$ is countable there exists a set $N$ of full measure such that on $N$ the sequences $\left\{\mu_{t}\left(B_{j}\right)\right\}_{t-1}^{\infty}$ all converge simultaneously. Thus $\mu_{\infty}$ is a probability measure and, on $N, \mu_{t}$ converges to $\mu_{\infty}$ in the topology of weak convergence.

Q.E.D.

\section{REFERENCES}

1. B. Allen, Utility perturbations and the equilibrium price set, J. Math. Econom. 8 (1982), $277-307$.

2. B. AlLEN, Generic existence of completely revealing equilibria for economies with uncertainty when prices convey information, Econometrica 49 (1981), 1173-1199.

3. B. Allen, Strict rational expectations equilibria with diffuseness, J. Econom. Theory 27 (1982), 20-46.

4. A. BejA, The limited information efficiency of market processes, Working Paper No. 43, Research Program in Finance, U. C. Berkeley, 1976.

5. T. BEwLEY, An integration of equilibrium theory and turnpike theory, Discussion Paper No. 405, Northwestern University, 1980.

6. P. Billingsley, "Convergence of Probability Measures," Wiley, New York, 1968. 
7. L. Blume, New techniques for the study of stochastic equilibrium process, J. Math. Econom. 9 (1982), 61-76.

8. L. Blume, M. Bray, AND D. EASLey, Introduction to the stability of rational expec tations equilibrium, J. Econom. Theory 26 (1982), 313-317.

9. L. Blume and D. Easley, Learning to be rational, J. Econom. Theory 26 (1982), 340-351.

10. L. Blume AND D. EAsley, Rational expectations equilibrium and the efficient markets hypothesis, unpublished working paper, March 1981.

11. M. Bray and D. Kreps, Rational learning and rational expectations, Research Paper No. 616, Graduate School of Business, Stanford University, August 1981.

12. J. L. Dоoв, Application of the theory of martingales, Colloq. Internat. CNRS (1947), $22-28$.

13. J. Harsanyi, Games with incomplete information played by bayesian players, Parts I, II, III, Management Sci. 14 (1967-1968), 159-182, 320-334, 486-502.

14. M. Hellwig, Rational expectations equilibrium with conditioning on past prices: A mean-variance example, J. Econom. Theory 26 (1982).

15. W. Hildenbrand, "Core and Equilibria of a Large Economy," Princeton Univ. Press, Princeton, N. J., 1974.

16. J. JORDAN, On the efficient markets hypothesis, Econometrica 51 (1983), 1325-1344.

17. J. JORDAN, The generic existence of rational expectations equilibria in the higher dimensional case. J. Econom. Theory 26 (1982), 224-243.

18. J. JORDAN AND R. RADNER, The nonexistence of rational expectations equilibrium: A robust example, Department of Economics, Unjversity of Minnesota, 1979.

19. J. JoRdAN AND R. RADNER, Rational expectations in microeconomic models: An overview, J. Econom. Theory 26 (1982), 201-223.

20. R. RADnER, Rational expectations equilibrium: Generic existence and the information revealed by prices, Econometrica 47 (1979), 655-678.

21. L. Schwartz, On bayes procedures, Z. Wahrsch. Verw. Gebiete 4 (1965), 10-26.

22. R. M. TownSEND, Market anticipations, rational expectations and bayesian analysis, Internat. Econom. Rev. 19 (1978), 481-494. 